\title{
Immunohistochemical analysis of ghrelin expression in various types of adrenal tumors
}

\author{
Hanna Komarowska ${ }^{1}$, Agnieszka Malinska ${ }^{2}$, Zhanat Komekbai ${ }^{3}$, Barbara Brominska ${ }^{1}$, \\ Katarzyna Bednarek-Rajewska ${ }^{4}$, Marek Ruchala ${ }^{1}$, Marcin Rucinski ${ }^{2}$
}

${ }^{1}$ Department of Endocrinology, Metabolism and Internal Medicine, Poznan University of Medical Sciences, Poznan, Poland

${ }^{2}$ Department of Histology and Embryology, Poznan University of Medical Sciences, Poznan, Poland

${ }^{3}$ West Kazakhstan Marat Ospanov Medical University, Aktobe, Kazakhstan

${ }^{4}$ Department of Clinical Pathology, Poznan University of Medical Sciences, Poznan, Poland

\begin{abstract}
Introduction. Ghrelin, originally isolated from the endocrine cells of the gastric mucosa, is also expressed in many peripheral tissues, including normal adrenals and adrenocortical tumors. It was shown that ghrelin stimulates proliferation and inhibits apoptosis of adrenocortical cells. In the current study, we compared ghrelin expression at the protein level in various adrenal tumors. We analyzed whether immunoreactive ghrelin could be considered as a potential marker for different types of adrenal tumors.

Material and methods. Study was carried out on 200 adrenal specimens arranged on microscope slide in tissue microarray format. We performed standardized immunohistochemical reactions with semiquantitative reaction intensity measurements.

Results. At the protein level, the expression of ghrelin was significantly reduced in adrenocortical adenocarcinoma in relation to the control group and pheochromocytoma as well as cancer-adjacent normal adrenal tissue. In contrast, a relatively high ghrelin expression was found in pheochromocytoma compared to all analyzed groups, with the exception of cancer-adjacent normal adrenal tissue.

Conclusions. The ghrelin expression profile at the protein level may be associated with the type of adrenal tumor. In this context, our results suggest that adrenal immunoreactive ghrelin may be considered as a sensitive and specific marker for differentiating adrenocortical carcinoma from adrenocortical adenoma and pheochromocytoma. (Folia Histochemica et Cytobiologica 2021, Vol. 59, No. 2, 86-94)
\end{abstract}

Key words: ghrelin; immunohistochemistry; adrenal tumors; adrenal cortex; adrenal medulla

\section{Introduction}

The management of adrenal tumors is quite challenging, mainly due to their growing frequency, diagnostic complexity, and limited availability of treatment option

\footnotetext{
Correspondence address: Hanna Komarowska, MD, PhD

Department of Endocrinology, Metabolism

and Internal Medicine, Poznan University of Medical Sciences,

Przybyszewskiego 49, 60-355 Poznan, Poland

e-mail: hkomar@ump.edu.pl

phone: +4861 869 1330; fax: 618691682
}

for adrenocortical carcinoma (ACC). In patients without premortem suspicion of adrenal disease, the prevalence of adrenal tumors at autopsy is found to be as high as $32 \%$. The occurrence of adrenal incidentaloma (AI) in computed tomography series is approximately $5 \%$ [1], whereas about $80 \%$ of cases are benign adenomas. More complex lesions such as adrenocortical carcinoma (ACC) or pheochromocytoma are rarely diagnosed at a rate of about $5 \%$ each, while $2 \%$ of AI turns out to be metastases [1,2]. Other lesions including ganglioneuroma, neuroblastoma, myelolipoma, cysts, or inflammatory tumors are detected occasionally. 
Most of the adrenal tumors remain hormonally inactive. However, depending on the level of hormonal activity, these tumors may cause various symptoms. Patients with active adrenal tumors or suspected malignancies are usually referred for adrenalectomy [1]

ACC is a rare, highly aggressive cancer. It has an incidence of about $0.7-2$ new cases per million per year. ACC is more frequently detected in 40-60 years old people [3]. The diameter of this tumor usually ranges from 5 to $20 \mathrm{~cm} \mathrm{[3].} \mathrm{ACC} \mathrm{is} \mathrm{diagnosed} \mathrm{using} \mathrm{Weiss}$ criteria with Aubert's modifications [4]. Pathological assessment is crucial for the correct diagnosis of ACC, but it is difficult to perform. Moreover, the frequency of misdiagnoses is relatively high. For instance, in a large consecutive series consisting of 300 cases tested in Italy, $26(13 \%)$ were misdiagnosed. The most common problem encountered in the diagnosis of ACC is the differentiation pheochromocytoma from ACC. Other diagnostic pitfalls include metastases from other primary cancers, primary tumors of adrenal soft tissue, and adrenal adenomas [5-7]. Due to these reasons, there is a high demand for the identification of ACC markers.

Ghrelin is the ligand of growth hormone secretagogue receptor type 1 (GHS-R1) [8]. It was first identified by Kojima et al. as a 28 -amino acid peptide, having an n-octanoyl moiety in serine 3 position [8]. Ghrelin is generated from the preproghrelin precursor during post-translational cleavage. Ghrelin is predominantly secreted by endocrine cells of the gastric mucosa, but its expression was also found in many other organs, including the small and large intestine, hypothalamus, pituitary, adrenal glands, testes, ovaries, thyroid gland, liver or lungs [8-14]. The main role of ghrelin is to regulate energy homeostasis and secretion of growth hormone. Thus far, many studies have confirmed the physiological and pathological effects of ghrelin on various organs [15-18]. Ghrelin and its receptors (GHS-R1a and GHS-R1b) were found in human adrenal glands [19]. Ghrelin does not influence the secretion of adrenal steroid hormones [20]. However, it increases the proliferation and decreases the apoptosis of adrenocortical cells [21, 22]. The ghrelin mRNA level in the human adrenal glands was evaluated in previous studies, using mainly the qPCR technique [9, 10, 23].

Currently, 13 alternative splice transcript variants of the ghrelin gene are described in the NCBI database; however, not all of them are translated into protein. Individual variants of the ghrelin gene also differ in the length of the $3^{\prime}$ and $5^{\prime}$ untranslated regions, which determine the stability of these transcripts [24, 25]. Therefore, it can be assumed that the expression of ghrelin at the mRNA level may not correlate with that at the protein level. Due to the aforementioned facts, our study aimed to compare the expression of ghrelin at the protein level in various adrenal tumors using the immunohistochemical (IHC) technique with a tissue microarray (TMA) containing 200 human adrenal samples. We also analyzed whether immunoreactive ghrelin can be considered as a potential marker for different types of adrenal tumors. To the best of our knowledge, this study is the first to evaluate the ghrelin protein level in a relatively large group of tissues derived from various adrenal tumors.

\section{Materials and methods}

Tissue material. The unstained adrenal gland disease spectrum (adrenal cancer progression) tissue microarray (TMA) slide (AD2081) was purchased from US Biomax, Inc. (Rockville, MD, USA). This histological slide contained 200 cores of adrenal gland tissue specimens, which included 20 samples of adrenocortical adenocarcinoma, 6 neuroblastoma, 2 ganglioneuroma, 60 pheochromocytoma, and 84 adrenocortical adenoma (ACA), in addition to 12 samples of normal tissue adjacent to ACC and 16 biopsy samples of normal adrenal tissue. Since we used the commercially available TMA slide, our study did not require the approval of local bioethics commission. Research complies with the Declaration of Helsinki.

Immunohistochemical analyses. For the IHC analyses, we employed the StreptABComplex/HRP method modified by biotinylated tyramine (Dako Catalysed Signal Amplification System, Peroxidase, K1500; Dako, Copenhagen, Denmark). Briefly, after deparaffinization, the sections were rehydrated in decreasing concentrations of ethanol and rinsed in phosphate-buffered saline (PBS). Antigen expression was enhanced by heat-induced epitope retrieval (HIER) treatment of tissue samples. The slides were placed in Target Retrieval Solution, Citrate pH 6.1 (S2369; Dako), steamed for $5 \mathrm{~min}$, and then allowed to cool to room temperature (RT) in the buffer for $20 \mathrm{~min}$. After rinsing in PBS, the endogenous activity of peroxidase was blocked by treating the sections with $10 \%$ hydrogen peroxide for $10 \mathrm{~min}$, while the nonspecific-binding sites were blocked by incubating with $10 \%$ t normal goat serum in PBS for $30 \mathrm{~min}$ at RT (ab7481: Abcam). Following this step, the sections were incubated with a mouse monoclonal anti-ghrelin antibody (1:100, ab57222; Abcam) overnight at $4^{\circ} \mathrm{C}$. Then, the tissue samples were rinsed and incubated with a biotinylated goat anti-mouse antibody (1:300; Dako) for $60 \mathrm{~min}$ at RT before incubation with streptavidin-horse radish peroxidase conjugate. The peroxidase reaction was developed using diaminobenzidine (S3000; Dako). Then, the specimens were lightly counterstained with Mayer's hematoxylin, dehydrated, and mounted. The sections incubated with nonimmune 
IgG1 (X 0931; Dako) as well as those in which the primary or secondary antibody was omitted were treated as negative controls. To determine the consistency of antibodies reactivity, commercially available serial sections obtained from the human gastric fundus (NBP2-30204; Novus Biologicals, Centennial, CO, USA) were used as external positive controls. The whole slide was digitalized with a Mirax-Midi slide scanner (Zeiss, Jena, Germany). The IHC staining was analyzed and documented at a high magnification using CaseViewer 2.3 (64-bit version) for Windows (3D Histech Ltd., Budapest, Hungary).

Semiquantitative evaluation of ghrelin protein expression. The expression of ghrelin protein was analyzed using the densitometric method. First, the blue-violet stain produced by hematoxylin was removed from the scanned slide, retaining only the brown stain of the appropriate IHC reaction, using Adobe Photoshop ver. 21.1.0 (Adobe Inc., San Jose, CA, USA). The image thus prepared was subjected to densitometric analysis using Image J software (Image J 1.5q, Wayne Rasband, National Institutes of Health, Bethesda, MD, USA) according to The Open Lab Book protocol (https://theolb.readthedocs.io/en/latest/imaging/measuring-cell-fluorescence-using-imagej.html) adapted to the TMA format. Using this software, the integrated density from each of the TMA specimens was calculated, with the fixed diameter covering 8800 pixels/specimen. The signal of the background was also determined and subtracted from that of the measured specimens.

Statistical analysis. All the statistical analyses were performed using the $\mathrm{R}$ programming language environment supported by the "ggplot2" library for graph drawing. Densitometric values determined from each of the studied groups were presented as a boxplot, with the values of median and interquartile range (IQR), and the densitometric data from individual patients were superimposed on the appropriate boxplots and displayed as dots. Comparisons between the groups were carried out using Kruskal-Wallis test followed by Dunn post hoc test. The differences between the groups were indicated using the letter annotation, where different letters indicate significant $(\mathrm{p}<0.05)$ differences between the compared groups. To evaluate the applicability of ghrelin as a potential biomarker for the differentiation of different types of adrenal tumors, we used the receiver operating characteristic (ROC) curve plot generated in the “pROC” library [26].

\section{Results}

To investigate the ghrelin's expression in various types of adrenal tumors and normal adrenal gland tissues, we used the commercially available adrenal gland disease spectrum (adrenal cancer progression) tissue array. The same experimental conditions were applied to all the samples, allowing for standardized IHC reactions and more reliable semiquantitative measurements. The expression analysis was carried out on 200 adrenal specimens described in the Material and Methods section. The general profile of the stained specimens is presented in Figure 1. Within the entire tissue array, the anti-ghrelin IHC staining of different intensities, ranging from relatively weak to strong, was observed.

To assess semiquantitatively expression of ghrelin at the protein level, we performed densitometric analysis using ImageJ software. The integrated density values obtained for individual patients assigned to appropriate groups are presented in Figure 2. Statistical analysis performed using the nonparametric Kruskal-Wallis test revealed a very low p-value ( $\mathrm{p}=$ 6.48e 13), indicating significant differences between the compared groups. The subsequent Dunn post hoc test showed that the expression of ghrelin at the protein level was significantly lower by $30 \%$ in adrenocortical adenocarcinoma (median [IQR] = 78.56 [80.2-65.1]) in relation to the control group (median $[\mathrm{IQR}]=110.87$ [118.45-99.44]) as well as both pheochromocytoma (median [IQR] $=133.4$ [147.56-109.77]) and cancer-adjacent normal adrenal tissue (median $[\mathrm{IQR}]=118.26$ [126.35-97.93]). A relatively low level of ghrelin expression was also observed in neuroblastoma (median [IQR] $=90.36$ [98-82.92]), compared to ACC, pheochromocytoma, and cancer-adjacent normal adrenal tissue. By contrast, a relatively high level of ghrelin expression was found in pheochromocytoma, in which the expression was significantly higher by $20 \%$ in relation to the control group. The expression of ghrelin in the pheochromocytoma was also significantly higher than all the other analyzed groups, except for cancer-adjacent normal adrenal tissue. Due to its small number, the ganglioneuroblastoma group was excluded from the statistical analyses.

Although no statistically significant difference was observed in ghrelin expression between the normal adrenal and cancer-adjacent normal adrenal tissue, it should be noted that there was a markedly stronger anti-ghrelin reaction in tumor-adjacent normal adrenal tissue with respect to tumor cells (Figs. 3G and $3 \mathrm{H}$ ), which confirmed the previously reported results. In the detailed analysis of ghrelin expression at high magnification, we observed a strong cytoplasmic expression of ghrelin within the adrenal cortex and chromaffin parenchymal cells compared to the relatively weakly stained connective tissue and vascular endothelium. Strong nuclear staining was observed in several ACA and pheochromocytoma samples. 


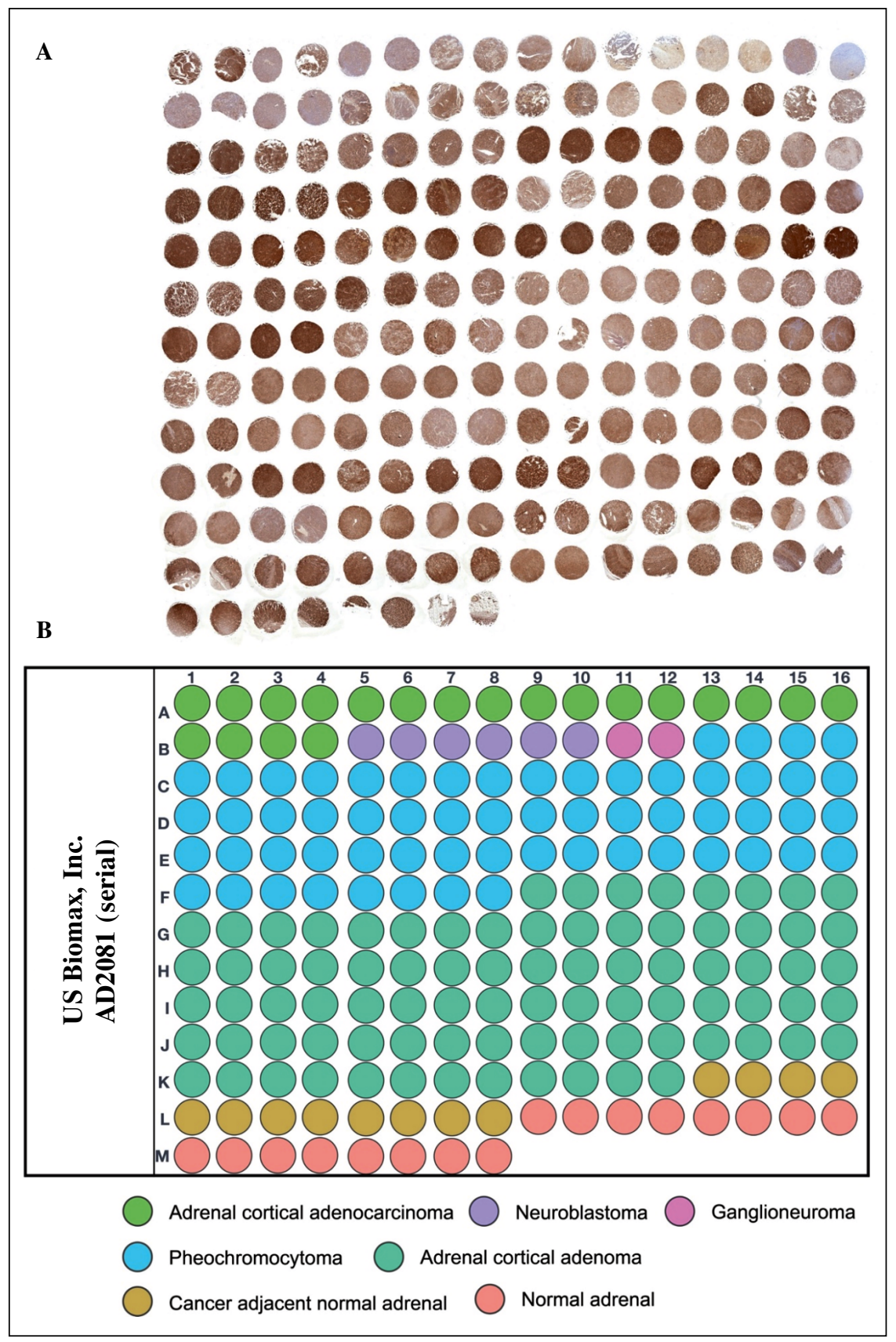

Figure 1. Analysis of ghrelin expression in adrenal gland disease spectrum (adrenal cancer progression) tissue microarray (TMA) slide. This histological slide contained 200 cores of adrenal gland tissue specimens, which included 20 samples of adrenocortical adenocarcinoma (ACC), 6 neuroblastoma, 2 ganglioneuroma, 60 pheochromocytoma, and 84 adrenocortical adenoma (ACA), and 12 samples of normal tissue adjacent to ACC and 16 biopsy samples of normal adrenal tissue. A. The general profile of the stained specimens. B. TMA map showing the relevant groups marked in appropriate colors.

The ROC curve analysis showed that ghrelin expression could be considered as a potential biomarker for differentiating ACC from normal adrenal tissue, and ACC from other adrenal tumors. The cut-off integrated density values used for differentiating between the compared groups were as follows: ACC vs. normal adrenal tissue $=91.7$ (specificity $95 \%$ and sensitivity $81.2 \%$ ), $\mathrm{ACC} v$ s. $\mathrm{ACA}=82.4$ (specificity $85 \%$ and sensitivity $92.9 \%$ ), and ACC vs. pheochromocytoma
$=91.7$ (specificity $95 \%$ and sensitivity $90 \%)$. All the compared groups were also characterized by a high area-under-curve (AUC) value as shown in Figure 4.

\section{Discussion}

Thus far, only a few studies have examined the expression of ghrelin in adrenal tumors [23, 27, 28]. In the study of Barzon et al., as well as our previous study [27, 


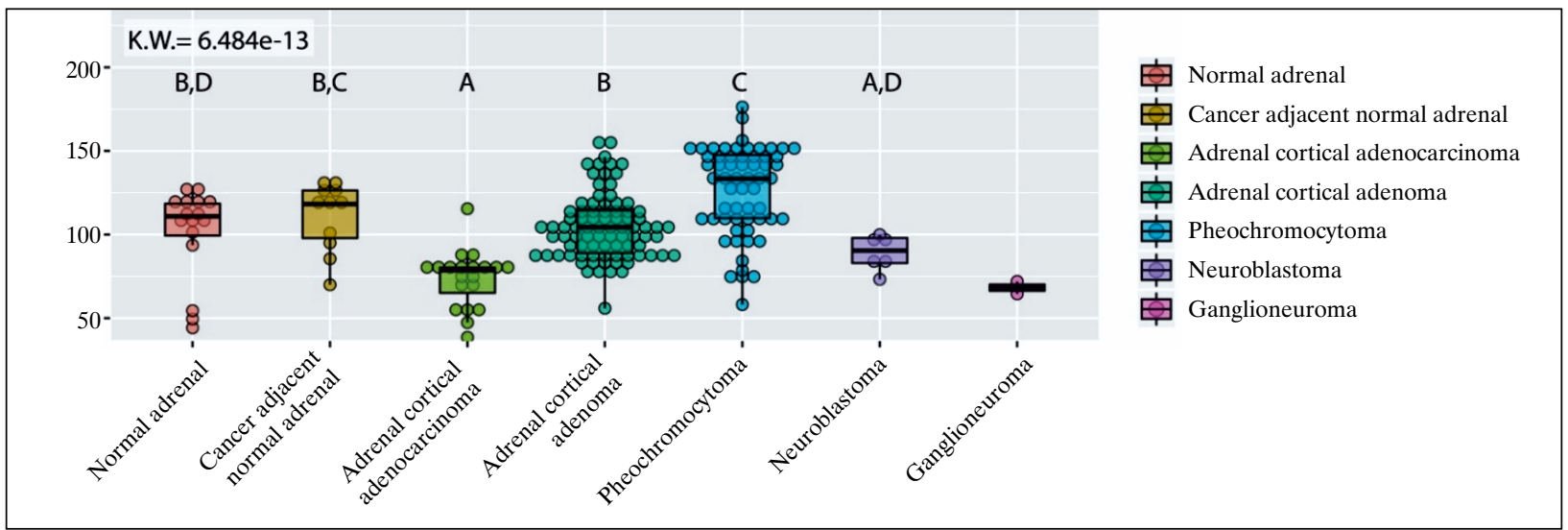

Figure 2. Densitometric analysis of ghrelin expression in adrenal gland disease spectrum (adrenal cancer progression) tissue array slide. Each of the groups was presented as a boxplot, with the values of median and IQR. Densitometric data from individual patients were superimposed on the appropriate boxplots and displayed as dots. Comparisons between the groups were carried out using Kruskal-Wallis followed by Dunn post hoc test. The differences between the groups were indicated using the letter annotation, where different letters indicate significant $(\mathrm{p}<0.05)$ differences between the compared groups.
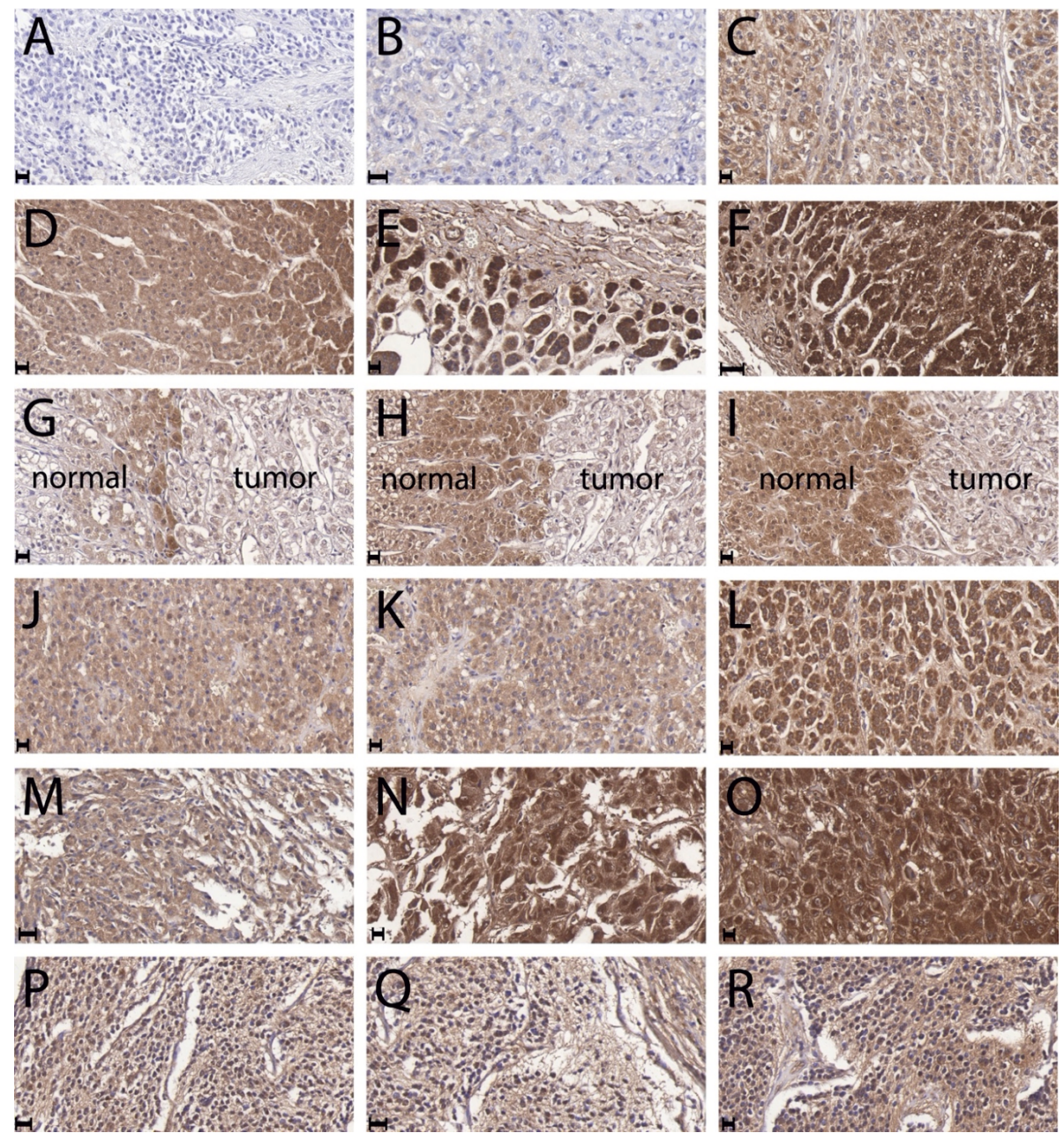

Figure 3. Representative images of ghrelin immunohistochemical reactivity in (A-C) ACC, (D-F) normal adrenal tissue, (G-I) tumor-adjacent normal tissue, (J-L) ACA, (M-O) pheochromocytoma, and (P-R) neuroblastoma. The left column shows the weakest expression from each group; the middle column presents the medium expression of the analyzed protein, and the right column shows the strongest expression. Abbreviations as for Figure 1. Scale bar: $20 \mu \mathrm{m}$. 

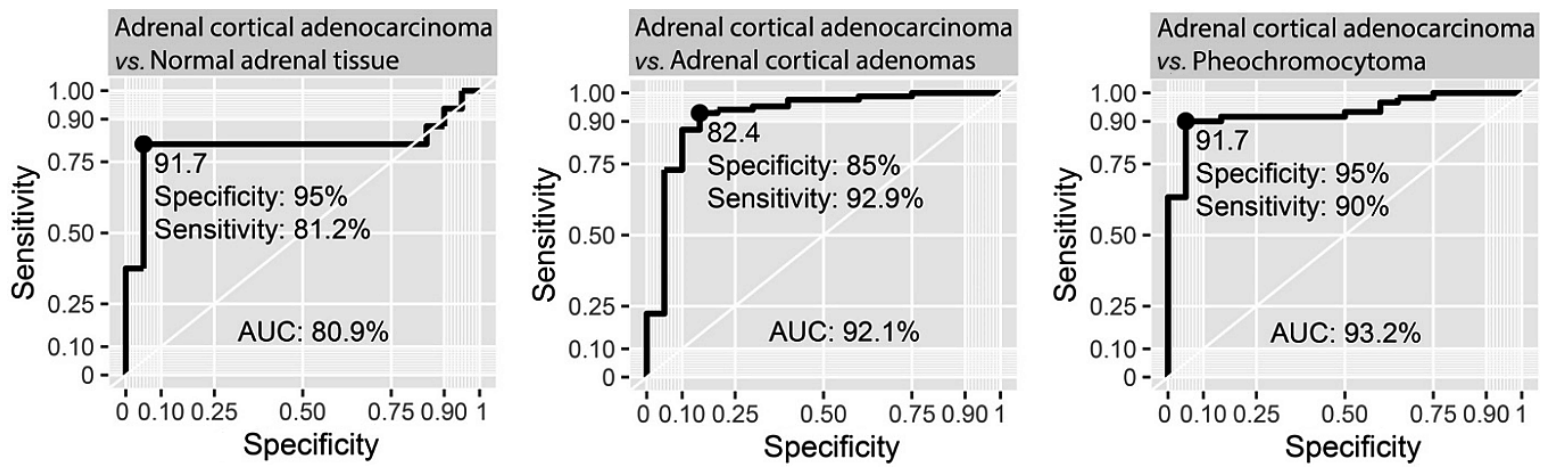

Figure 4. ROC analysis of the use of ghrelin immunoreactivity as a biomarker for differentiating ACC from normal adrenal tissue, ACC from ACA, and ACA from pheochromocytoma. The cut-off values used for differentiation, specificity, sensitivity, and the AUC values are shown in each of the graphs. Abbreviations as for Figure 1.

28], ghrelin expression was tested only at the mRNA level. Ueberberg et al. [23] investigated the expression of ghrelin at the level of both mRNA and protein in adrenal adenoma, pheochromocytoma, and healthy adrenal tissue, while Raghay et al. [10] studied its expression in the adrenal medulla and pheochromocytoma. To our knowledge, our present study is the first in which the expression of ghrelin at the protein level was evaluated in ACC compared with other tumors derived from the adrenal cortex or medulla. Ghrelin is synthesized in normal human adrenal glands [23]. This result was also confirmed in our current study; however, we found low immunoreactivity of ghrelin protein in ACC compared with normal adrenals, ACA, and pheochromocytoma. Based on the ROC curves, we suggest that ghrelin may serve as a potential immunohistochemical marker of ACC due to its high sensitivity and specificity. It should be noted that during the histopathological examination, the diagnosis of ACC can be very difficult. In such a scenario, ghrelin immunostaining may form a valuable prognostic marker for distinguishing malignant and aggressive ACC from benign ACA and pheochromocytoma.

Various hypotheses can be proposed to explain the low expression of ghrelin in ACC. First, there may be a mutation in the ghrelin gene that would cause disturbances in the protein expression. If the high immunoreactivity of ghrelin is assumed to be due to the presence of binding sites (i.e. ghrelin receptors) in the examined tissues, then the low immunoreactivity could be linked to a decrease in the number of receptors - negative feedback-based defense downregulation.

Furthermore, ghrelin acts as a stimulatory factor for adrenocortical proliferation [20-23, 28]. Therefore, it can be assumed that locally produced ghrelin could stimulate adrenal tumor cell proliferation via auto or paracrine way. However, due to the low expression of ghrelin in ACC this mechanism appears unlikely.

The role of ghrelin splice variants in carcinogenesis was also previously described [29]. Different posttranslational cleavage sites of ghrelin precursor may lead to the formation of new peptides with other functions. Thus, one of the splice variants that our IHC method has not identified might be involved in the etiopathogenesis of ACC.

In our previous work [28], we evaluated the mRNA expression of ghrelin in adrenal tumors. However, our current results are quite surprising - the mRNA expression of ghrelin was the highest and the protein expression was the lowest in ACC. Furthermore, in pheochromocytomas, we found low mRNA expression, whereas the highest protein expression. Nonetheless, our results were not unpredictable. Ghelardoni et al. compared the gene and protein expression of ghrelin in different human tissues and observed that the protein expression was discordant with the gene expression. The authors suggested that different tissues may significantly differ in their rate of ghrelin mRNA translation or posttranslational events [11]. The regulation of posttranscriptional and translational processes and protein degradation can contribute as much to variations in protein concentration as transcription and transcript degradation [30].

Perhaps, the low expression of ghrelin protein results from the epigenetic regulation of gene expression by circulating microRNAs (miRNAs). miRNAs are small (30-32)-nucleotide noncoding RNA that act as regulators of posttranscriptional stability and/ /or translation of RNA (mRNA) [31]. Certain types of miRNAs are more expressed in ACC tissues than in ACA [31-33]. It is assumed that miRNAs might 
interfere with translation or enhance the degradation of ghrelin.

Alternative splicing of preproghrelin mRNA is complicated and can lead to the formation of many variants of mRNA and, consequently, proteins. Over a dozen transcript variants of the human preproghrelin gene exist, but it is not known if they are all transcribed into protein. The lack of relationship between RNA expression and protein formation may be due to changes in processing during translation, post-translation, and protein degradation. New peptides resulting from alternative splicing may perform different functions than ghrelin. For instance, in breast cancer, the ghrelin variant In1-ghrelin was reported to be overexpressed and to promote basal proliferation in the breast cancer cell line [32]. In addition, an increase in the risk of breast cancer was associated with the polymorphisms of ghrelin gene [29].

Ghrelin gene-derived splice variants are overexpressed in breast cancer, which suggests that an imbalance in the ghrelin system's regulation may be associated with the pathogenesis of breast tumor [33]. Perhaps, a similar mechanism is involved in the development of adrenal tumors.

We can also assume that if low mRNA expression is associated with high protein levels, we are dealing with a very stable transcript, which implies that a small amount of transcripts will give us a large amount of protein. On the other hand, if there are many unstable, easily degraded transcripts, the protein level will remain low.

An additional interesting observation is the nuclear ghrelin immunostaining in ACC. Similar reactions have previously been observed in human normal and polycystic ovaries and in the rat testes [12, 34]. The authors conclude that the nuclear expression of ghrelin suggests its particular role in the intracellular regulation, where ghrelin could control the expression of other genes, acting as a specific transcription factor [35].

Thus far, no data have been published regarding the IHC analysis of ghrelin in ACC, but our observations are in line with the studies on different cancer types. Aydin et al. evaluated the immunoreactivity of ghrelin in gastric adenocarcinoma and mucoepidermoid carcinoma of the salivary glands. They found that negative ghrelin immunostaining can differentiate tumors from normal tissues [36]. Similarly, in renal cell carcinoma and oral squamous cell carcinoma, the ghrelin protein expression was frequently lower than that in normal tissues or was even absent $[37,38]$. Ghrelin expression has also been associated with better outcomes in various malignancies. For example, patients with breast cancer expressing ghrelin have a lower risk of death compared to those lacking ghrelin expression in tumors [33]. It seems that reduced ghrelin immunoexpression is associated with an increased risk of malignancy of some lesion.

Data on the expression of ghrelin protein in pheochromocytoma are scared. In the present study, we showed that ghrelin expression was significantly lower in ACC than pheochromocytoma. Ueberberg et al. investigated ghrelin expression in 43 ACA (17 functional and 16 nonfunctional) and 13 pheochromocytomas. They found that ghrelin protein was expressed in $54-87 \%$ of ACA and $0 \%$ of pheochromocytoma samples tested [23]. In turn, Raghay et al. evaluated the expression of ghrelin protein using two types of antibodies - goat and rabbit [10]. The authors used goat antibody to evaluate ghrelin immunoexpression in both human normal medulla and pheochromocytomas. Ghrelin immunoexpression was observed in the medulla. Chromaffin cells were immunostained with ghrelin, whereas the neurons were negative. The five human pheochromocytomas studied showed positivity for goat anti-ghrelin with focal or diffuse immunostaining patterns. The intensity of immunoreactivity varied between different tumors and also between different cells of the same tumor. However, when rabbit antibodies were used, no reaction was found in the normal medulla and pheochromocytomas. The authors suggested that the difference could be due to the fact that rabbit antibody can recognize only the mature peptide form having 28 amino acids, whereas goat antibody can detect all ghrelin forms - precursors, proghrelin, and mature form [10]. Ueberberg et al. used rabbit antibody while we performed tests with a goat antibody, which may be the reason for the different results observed in the case of pheochromocytomas. Both results suggest that in pheochromocytoma, mature ghrelin is not produced or is rapidly degraded. Pheochromocytoma is the only adrenal tumor in which the effect of ghrelin on the secretory activity of the tumor has been confirmed [39, 40]. Nanmoku et al. observed stimulatory ghrelin's effect on dopamine secretion in rat pheochromocytoma PC 12 cells [40]. It is unlikely that ghrelin directly affects the production and secretion of adrenocortical hormones [20], but some reports suggest that ghrelin stimulates dopamine secretion in pheochromocytomas [40]. The difference associated with protein expression in pheochromocytomas, may be related to its effect on dopamine secretion.

In our study, the lowest immunoexpression of ghrelin protein was noted in ganglioneuroblastoma, which might be attributed to the origin of this tumor. However, due to the small size of this group, the data 
obtained are difficult to be discussed. There no data on this subject in the available literature.

In conclusion, the histopathological diagnosis of ACC is often difficult. Our research suggests that ghrelin immunoreactivity may be considered as a sensitive and specific marker for differentiating ACC from ACA and pheochromocytoma. However, further research is required to determine the causes of differential ghrelin expression in adrenal tumors.

\section{Financial disclosure}

This research was supported by "Opus Grant" program of the National Science Center No. UMO-2017/25/B/ /NZ4/00065. Zhanat Komekbai (WKMOMU) \& Agnieszka Malinska (PUMS) were supported by the Social Health Insurance Project, Republic of Kazakhstan (Contract No. SHIP-2.3/CS-02).

\section{Author Contribution}

Conceptualization, HK, AM and Marcin R; Data curation, HK, BB, K B-R and Marek R; Formal analysis, Marcin R; Funding acquisition, $\mathrm{AM}$ and $\mathrm{ZK}$; Investigation, HK, BB and K B-R; Methodology, HK, ZK and Marcin R; Project administration, AM and ZK; Resources, AM and ZK; Software, Marcin R; Supervision, HK, AM and Marcin R; Validation, HK, AM and Marek R; Visualization, HK, BB and K B-R; Writing - original draft, HK, BB and K B-R; Writing - review \& editing, HK, AM, ZK, Marek R and Marcin R. All authors approved the version to be published and agreed to be accountable for all aspects of the work in ensuring that questions related to the accuracy or integrity of any part of the work are appropriately investigated and resolved.

\section{Conflicts of interest}

The authors declare no conflict of interest.

\section{References}

1. Dietrich CF, Correas JM, Dong Yi, et al. WFUMB position paper on the management incidental findings: adrenal incidentaloma. Ultrasonography. 2020; 39(1): 11-21, doi: 10.14366/usg.19029, indexed in Pubmed: 31786909.

2. Lenders JWM, Eisenhofer G. Update on modern management of pheochromocytoma and paraganglioma. Endocrinol Metab (Seoul). 2017; 32(2): 152-161, doi: 10.3803/ EnM.2017.32.2.152, indexed in Pubmed: 28685506.

3. Fassnacht M, Dekkers O, Else T, et al. European Society of Endocrinology Clinical Practice Guidelines on the management of adrenocortical carcinoma in adults, in collaboration with the European Network for the Study of Adrenal Tumors. Eur J Endocrinol. 2018; 179(4): G1-G46, doi: 10.1530/ EJE-18-0608, indexed in Pubmed: 30299884.

4. Aubert S, Wacrenier A, Leroy X, et al. Weiss system revisited: a clinicopathologic and immunohistochemical study of
49 adrenocortical tumors. Am J Surg Pathol. 2002; 26(12): 1612-1619, doi: 10.1097/00000478-200212000-00009, indexed in Pubmed: 12459628.

5. Duregon E, Volante M, Bollito E, et al. Pitfalls in the diagnosis of adrenocortical tumors: a lesson from 300 consultation cases. Hum Pathol. 2015; 46(12): 1799-1807, doi: 10.1016/j. humpath.2015.08.012, indexed in Pubmed: 26472162.

6. Komarowska H, Bednarek-Rajewska K, Kański M, et al. Epithelioid angiomyolipoma mimicking adrenal cortical carcinoma: A diagnostic pitfall. Oncol Lett. 2015; 10(4): 2130-2134, doi: 10.3892/ol.2015.3543, indexed in Pubmed: 26622807.

7. Komarowska H, Bromińska B, Janicka-Jedyńska M, et al. Adrenal Incidentaloma: Nothing Is Ever as It Seems. Am J Med. 2020; 133(9): 1048-1050, doi: 10.1016/j.amjmed.2019.12.039, indexed in Pubmed: 32001227.

8. Kojima M, Hosoda H, Date Y, et al. Ghrelin is a growth-hormone-releasing acylated peptide from stomach. Nature. 1999; 402(6762): 656-660, doi: 10.1038/45230, indexed in Pubmed: 10604470

9. Ueberberg B, Unger N, Saeger W, et al. Expression of ghrelin and its receptor in human tissues. Horm Metab Res. 2009; 41(11): 814-821, doi: 10.1055/s-0029-1233462, indexed in Pubmed: 19670151.

10. Raghay K, García-Caballero T, Bravo S, et al. Ghrelin localization in the medulla of rat and human adrenal gland and in pheochromocytomas. Histol Histopathol. 2008; 23(1): 57-65, doi: 10.14670/HH-23.57, indexed in Pubmed: 17952858.

11. Ghelardoni S, Carnicelli V, Frascarelli S, et al. Ghrelin tissue distribution: comparison between gene and protein expression. J Endocrinol Invest. 2006; 29(2): 115-121, doi: 10.1007/ BF03344083, indexed in Pubmed: 16610236.

12. Komarowska H, Waśko R, Iwanik K, et al. Ghrelin ovarian cell expression in patients with polycystic ovary syndrome: an immunohistochemical evaluation. Horm Metab Res. 2006; 38(12): 783-788, doi: 10.1055/s-2006-956506, indexed in Pubmed: 17163351.

13. Gnanapavan S, Kola B, Bustin SA, et al. The tissue distribution of the mRNA of ghrelin and subtypes of its receptor, GHS-R, in humans. J Clin Endocrinol Metab. 2002; 87(6): 2988, doi: 10.1210/jcem.87.6.8739, indexed in Pubmed: 12050285.

14. Andrusiewicz M, Komarowska H, Skibińska I, et al. Expression of ghrelin and ghrelin functional receptor GHSR1a in human pituitary adenomas. Pol Arch Intern Med. 2017; 127(3): 163-169, doi: 10.20452/pamw.3967, indexed in Pubmed: 28377557.

15. Komarowska H, Jaskula M, Stangierski A, et al. Influence of ghrelin on energy balance and endocrine physiology. Neuro Endocrinol Lett. 2012; 33(8): 749-756, indexed in Pubmed: 23391977.

16. Akalu Y, Molla MD, Dessie G, et al. Physiological Effect of Ghrelin on Body Systems. Int J Endocrinol. 2020; 2020: 1385138, doi: 10.1155/2020/1385138, indexed in Pubmed: 32565790 .

17. Tokudome T, Otani K, Miyazato M, et al. Ghrelin and the heart. Peptides. 2019; 111: 42-46, doi: 10.1016/j.peptides.2018.05.006, indexed in Pubmed: 29791869.

18. Angel CZ, Iguacel I, Mullee A, et al. Appetite-regulating hormones-leptin, adiponectin and ghrelin-and the development of prostate cancer: a systematic review and exploratory meta-analysis. Prostate Cancer Prostatic Dis. 2020; 23(1): 11-23, doi: 10.1038/s41391-019-0154-1, indexed in Pubmed: 31147627.

19. Carraro G, Albertin G, Abudukerimu A, et al. Growth hormone secretagogue receptor subtypes $1 \mathrm{a}$ and $1 \mathrm{~b}$ are ex- 
pressed in the human adrenal cortex. Int J Mol Med. 2004; 13(2): 295-298, doi.org/10.3892/ijmm.13.2.295, indexed in Pubmed: 14719137

20. Andreis P, Malendowicz L, Trejter M, et al. Ghrelin and growth hormone secretagogue receptor are expressed in the rat adrenal cortex: evidence that ghrelin stimulates the growth, but not the secretory activity of adrenal cells. FEBS Letters. 2003; 536(1-3): 173-179, doi: 10.1016/s00145793(03)00051-6.

21. Mazzocchi G, Neri G, Rucinski M, et al. Ghrelin enhances the growth of cultured human adrenal zona glomerulosa cells by exerting MAPK-mediated proliferogenic and antiapoptotic effects. Peptides. 2004; 25(8): 1269-1277, doi: 10.1016/j.peptides.2004.05.011, indexed in Pubmed: 15350694.

22. Delhanty PJD, van Koetsveld PM, Gauna C, et al. Ghrelin and its unacylated isoform stimulate the growth of adrenocortical tumor cells via an anti-apoptotic pathway. Am J Physiol Endocrinol Metab. 2007; 293(1): E302-E309, doi: 10.1152/ ajpendo.00377.2006, indexed in Pubmed: 17405826.

23. Ueberberg B, Unger N, Sheu SY, et al. Differential expression of ghrelin and its receptor (GHS-R1a) in various adrenal tumors and normal adrenal gland. Horm Metab Res. 2008; 40(3): 181-188, doi: 10.1055/s-2007-1004574, indexed in $\mathrm{Pu}-$ bmed: 18246525 .

24. Hosoda H, Kojima M, Matsuo $\mathrm{H}$, et al. Purification and characterization of rat des-Gln14-Ghrelin, a second endogenous ligand for the growth hormone secretagogue receptor. J Biol Chem. 2000; 275(29): 21995-22000, doi: 10.1074/jbc. M002784200, indexed in Pubmed: 10801861.

25. Seim I, Jeffery PL, Thomas PB, et al. Multi-species sequence comparison reveals conservation of ghrelin gene-derived splice variants encoding a truncated ghrelin peptide. Endocrine. 2016; 52(3): 609-617, doi: 10.1007/s12020-015-0848-7, indexed in Pubmed: 26792793.

26. Robin X, Turck N, Hainard A, et al. pROC: an open-source package for $\mathrm{R}$ and $\mathrm{S}+$ to analyze and compare ROC curves. BMC Bioinformatics. 2011; 12: 77, doi: 10.1186/1471-210512-77, indexed in Pubmed: 21414208.

27. Barzon L, Pacenti M, Masi G, et al. Loss of growth hormone secretagogue receptor 1a and overexpression of type $1 \mathrm{~b}$ receptor transcripts in human adrenocortical tumors. Oncology. 2005; 68(4-6): 414-421, doi: 10.1159/000086983, indexed in Pubmed: 16020971

28. Komarowska H, Rucinski M, Tyczewska M, et al. Ghrelin as a potential molecular marker of adrenal carcinogenesis: In vivo and in vitro evidence. Clin Endocrinol (Oxf). 2018; 89(1): 36-45, doi: 10.1111/cen.13725, indexed in Pubmed: 29682767.

29. Dossus L, McKay JD, Canzian F, et al. Polymorphisms of genes coding for ghrelin and its receptor in relation to anthropometry, circulating levels of IGF-I and IGFBP-3, and breast cancer risk: a case-control study nested within the European Prospective Investigation into Cancer and Nutrition (EPIC).
Carcinogenesis. 2008; 29(7): 1360-1366, doi: 10.1093/carcin/ bgn083, indexed in Pubmed: 18375957.

30. Vogel C, Marcotte EM. Insights into the regulation of protein abundance from proteomic and transcriptomic analyses. Nat Rev Genet. 2012; 13(4): 227-232, doi: 10.1038/nrg3185, indexed in Pubmed: 22411467.

31. Ambros V. The functions of animal microRNAs. Nature. 2004; 431(7006): 350-355, doi: 10.1038/nature02871, indexed in Pubmed: 15372042.

32. Gahete MD, Córdoba-Chacón J, Hergueta-Redondo M, et al. A novel human ghrelin variant (In1-ghrelin) and ghrelin-O-acyltransferase are overexpressed in breast cancer: potential pathophysiological relevance. PLoS One. 2011; 6(8): e23302, doi: 10.1371/journal.pone.0023302, indexed in Pubmed: 21829727.

33. Grönberg M, Nilsson C, Markholm I, et al. Ghrelin expression is associated with a favorable outcome in male breast cancer. Sci Rep. 2018; 8(1): 13586, doi: 10.1038/s41598-018-31783-x, indexed in Pubmed: 30206250.

34. Łukaszyk A, Rafińska L, Sawiński P, et al. Immunohistochemical and hybridocytochemical study on ghrelin signalling in the rat seminiferous epithelium. Folia Histochem Cytobiol. 2009; 47(3): 415-423, doi: 10.2478/v10042-009-0097-9, indexed in Pubmed: 20164026.

35. Buyse J, Janssen S, Geelissen S, et al. Ghrelin modulates fatty acid synthase and related transcription factor mRNA levels in a tissue-specific manner in neonatal broiler chicks. Peptides. 2009; 30(7): 1342-1347, doi: 10.1016/j.peptides.2009.04.015, indexed in Pubmed: 19409434.

36. Aydin S, Ozercan IH, Dagli F, et al. Ghrelin immunohistochemistry of gastric adenocarcinoma and mucoepidermoid carcinoma of salivary gland. Biotech Histochem. 2005; 80(34): 163-168, doi: 10.1080/10520290500387847, indexed in Pubmed: 16298902.

37. Dagli AF, Aydin S, Karaoglu A, et al. Ghrelin expression in normal kidney tissue and renal carcinomas. Pathol Res Pract. 2009; 205(3): 165-173, doi: 10.1016/j.prp.2008.10.002, indexed in Pubmed: 19054628.

38. Alnema MM, Aydin S, Ozkan Y, et al. Ghrelin and obestatin expression in oral squamous cell carcinoma: an immunohistochemical and biochemical study. Mol Cell Biochem. 2010; 339(1-2): 173-179, doi: 10.1007/s11010-009-0381-1, indexed in Pubmed: 20047070.

39. Obara-Moszyńska M, Kedzia A, Chmielnicka-Kopaczyk M. Expression of ghrelin in human fetal adrenal glands and paraadrenal nerve ganglions. Folia Histochem Cytobiol. 2009; 47(1): 25-28, doi: 10.2478/v10042-009-0023-1, indexed in Pubmed: 19419933.

40. Nanmoku T, Takekoshi K, Ishi K, et al. Effect of Ghrelin on catecholamine secretion in rat pheochromocytoma PC12 cells. Endocr Res. 2003; 29(1): 17-21, doi: 10.1081/erc-120018673, indexed in Pubmed: 12665315.

Submitted: 26 October, 2020

Accepted after reviews: 23 March, 2021 Available as AoP: 9 April, 2021 